\title{
Über \\ zwei eigenartige Gynandromorphe des Schwammspinners Lymantria dispar L.
}

Von Richard Goldschmidt (Berlin-Dahlem) und J. Machida (Tokyo).

Hierzu Tafel 3 und 4 Textfiguren.)

(Eingegangen am 20. November 1921.)

Es liegt in der Literatur eine solche Fülle von Beschreibungen gynandromorpher Insekten vor (s. die neuesten Zusammenfassungen von Cockayne und Morgan), daß ihre Vermehrung nur dann gerechtfertigt werden kann, wenn Objekte gefunden werden, die von mehr als kasuistischem Interesse sind. Die im folgendem zu beschreibenden beiden Stücke haben in der Tát einige Eigenschaften, die ein beträchtliches entwicklungsphysiologisches Interesse beanspruchen. Sie wurden von den beiden Verfassern in ihren Zuchten erhalten, ohne daß eine Ursache für das Erscheinen bekannt wäre. Beides sind bilaterale Gynandromorphe (Halbseitenzwitter), eine Bezeichnung, die aber nicht für alle Einzelheiten zutrifft. Im folgenden sind die von den beiden Autoren gefundenen Stücke als Gyn. G. und Gyn. M. unterschieden.

\section{Einzelbeschreibung.}

1. Herkunft. Gyn. G. erschien in einer sonst völlig normalen $\mathrm{F}_{1}$-Zucht aus den Rassen Tokyo $ᄋ \times$ Hokkaido $^{1}$ ) $\sigma^{7}$ neben 42 ㅇ $440^{7}$.

Gyn. M. erschien in einer $\mathrm{F}_{4}$-Zucht aus der Kreuzung der gleichen Rassen neben 33 o und $270^{\circ}$. Weder in diesen noch anderen Kombinationen war in zwölfjähriger Zucht mit sicher mehr als $100000 \mathrm{In}-$ dividuen jemals ein Gynandromorph erschienen. Über die Ursache des Auftretens ist nichts bekannt; es ist immerhin bemerkenswert, daß in

1) Wegen der Bedeutung dieser Rassen siehe Goldschmidt 1920a. Induktive Abstammungs- und Vererbungslehre. XXVIII. 
beiden Fällen Kreuzungen der Rassen Tokyo und Hokkaido involviert waren. Beide Individuen sind rechts $0^{x}$ und links $q$.

2. Benehmen. Der Gyn. G. war aus einer Puppe geschlüpft, die als $\sigma^{7}$ registriert worden war, und machte bei oberflächlicher Betrachtung auch den Findruck eines $\sigma^{7}$. Er benahm sich auch mehr wie ein $0^{7}$, d. h. er versuchte bei Reizung zu fliegen, brachte es aber nur bis zu einem unvollkommenen Flattern. Dabei arbeiteten die beiden Körperhälften ganz harmonisch und nicht etwa so als wenn eine "psychische" Trennung der beiden Körperhälften vorhanden gewesen wäre. Ein Kopulationsdrang fehlte beiden Geschlechtern gegenüber, auch wurde kein Versuch gemacht, die reichlich vorhandene weibliche Afterwolle abzureiben. Gyn. M. dagegen, der auch äußerlich zunächst wie ein ㅇ wirkt, benahm sich auch wie ein $q$, aber es kopulierte ebenfalls nicht. Dagegen rieb es sich nach einigen Tagen Afterwolle ab.

\section{3. Äußere Organisation.}

a) Der Kopf. Bei dem Gyn. G. ist die Farbe des Kopfes anf Scheitel und Stirn rechts männlich (braun), links weiblich (weiß) (s. Fig. 3, Taf. 3). Die rechte Antenne ist männlich, die linke weiblich. Die Labialtaster, die beim o dunkler sind als beim $0^{x}$, sind ziemlich gleichartig von männlicher Farbe. Auch bei dem Gyn. M. ist die Farbe des Kopfes rechts männlich und links weiblich, und ebenso auch die Antennen (s. Fig. 2, 4, Taf. 3). Bei ihm folgen aber auch die Lippentaster der Einteilung in rechts weiblich und links männlich.

b) Das Abdomen. In beiden Individuen hat es Umfang und Form eines weiblichen Abdomens (Fig. 2, 4, 6, 7, Taf. 3). In beiden Individuen ist es nach rechts (der männlichen Seite) gebogen, was zeigt, daß das Wachstum des Abdomens rechts männlich und links weiblich war. Die Farbe des Abdomens ist bei Gyn. G. männlich, wobei der dunkle Rückenstreif nicht fehlt. Gyn. M. hat ein weiblich gefärbtes, weißes Abdomen, aber auf der Banchseite ist die männliche Seite doch dunkler. Die Behaarung des Abdomens ist beim Gyn. G. mehr männlich, aber auf der weiblichen Seite ist Afterwolle vorhanden. Beim Gyn. M. ist sie ganz weiblich.

c) Der Thorax. Behaarung und Farbe des Thorax ist bei Gyn. G. männlich, bei Gyn. M. weiblich. Die Beine dagegen sind bei beiden Individuen rechts männlich, links weiblich, was sich hauptsächlich darin zeigt, daß tibia und tarsus des zweiten und dritten Beines auf der weiblichen Seite schwarze, auf der männlichen helle Behaarung zeigen (Fig. 4, Taf, 3). 
d) Die Flügel. Das Verhalten der Flügel der beiden Gynandromorphen ist besonders bemerkenswert (Fig. 1, 2, 3, 5, Taf. 3). Gyn. G.: Die Form der Flügel ist beiderseits nahezu männlich. Die Symmetrie ist aber keine vollständige, vielmehr sind die Flügel auf der weiblichen Seite ein wenig schlanker und länger, wodurch sie sich ein wenig dem weiblichen Zustand nähern. Die Farbe und Beschuppung der Flügel ist rechts völlig -männlich; links ist sie aber vorwiegend männlich mit

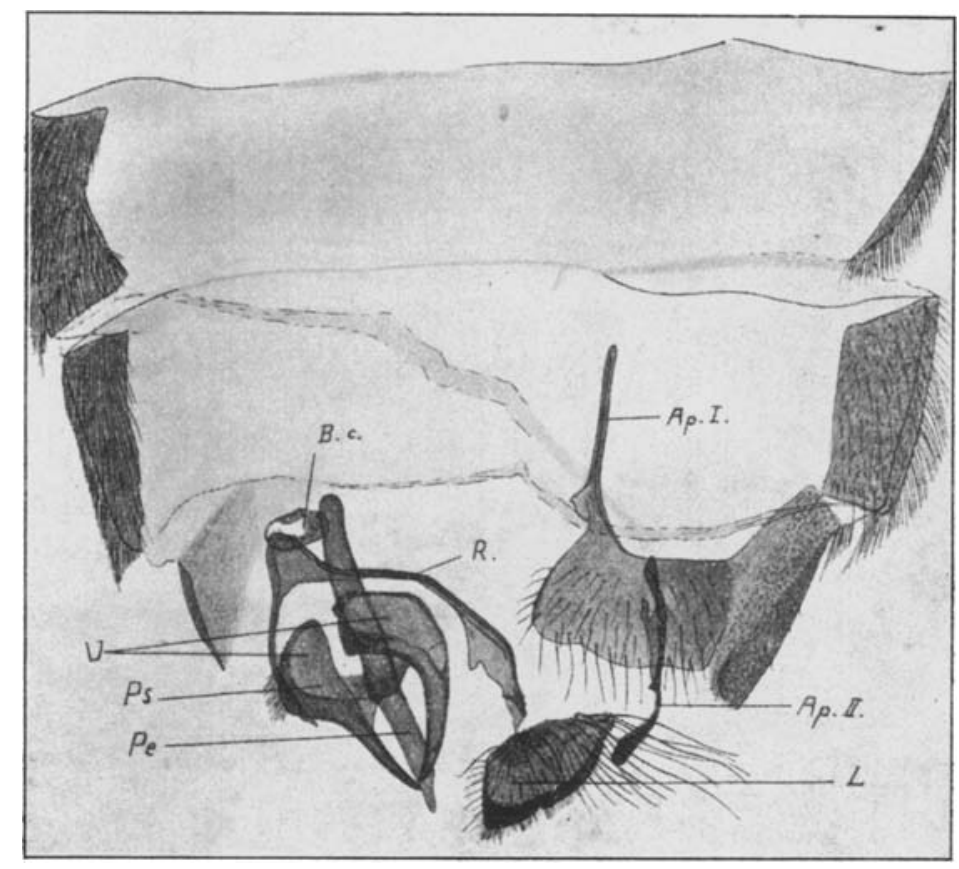

Textfig. A. Mazerierter Kopulationsapparat des Gyn. G.

unregelmäBig eingestreuten weiblichen Teilen (Fig. 1, 3, Taf. 3). Auf der Oberseite von Vorder- und Hinterflügel sind es unregelmäßige weiße Streifen und Keile, die aus dem braunen Untergrund hervortreten. Auf der Unterseite ist der größere Teil des Vorderflügels von weiblicher Farbe. Die von den Systematikern als Unterscheidungsmerkmal der Geschlechter besonders geschätzte Haftborste (frenulum) der Hinterflügelunterseite, die in eine Führung (retinaculum) des Vorderflügels paßt, ist auf beiden Seiten rein männlich. Gyn. M.: Die Flügelform ist rechts und links beträchtlich verschieden, indem die Flügel der 
männlichen Seite kleiner sind. Trotzdem ist ihre Form nicht rein männlich sondern nähert sich mehr der länglichen weiblichen Form. Die Flügelfarbe ist auf beiden Seiten weiblich, aber auf der männlichen Seite liegen nahe den Flügelspitzen Gruppen brauner männlicher Schuppen! Das frenulum ist links weiblich und rechts männlich.

4. Das Kopulationsorgan. Bei beiden Individuen finden sich rechts Teile des männlichen, links Teile des weiblichen Kopulationsorgans. (Beschreibung des normalen Kopulationsorgans s. Goldschmidt 1920). Gyn. G. (Textfig. A): rechts

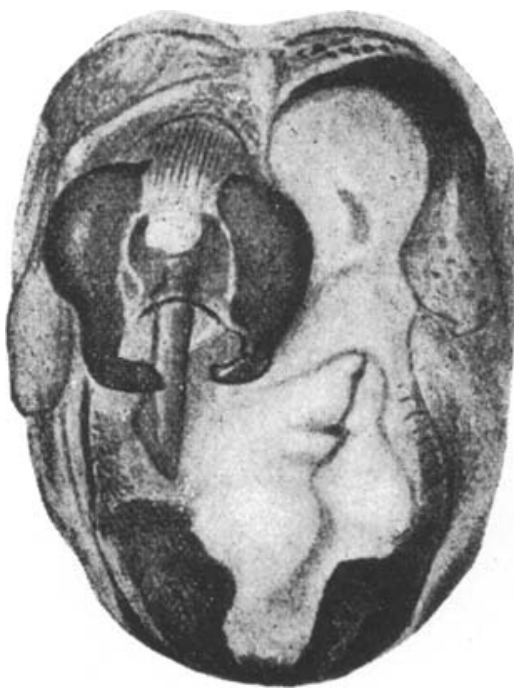

Textfig. B. Kopulationsorgane des Gyn. M ohne Mazeration. liegt ein männlicher Kopulationsapparat mit Penis $(\mathrm{Pe})$, Penisscheide (Ps) und den beiden symmetrischen Valven (V). Vom Segmentring $R$ ist etwa $2 / 3$ vorhanden, der Rest mit dem Uncus fehlt. Auf der linken Seite liegt genau ein halber weiblicher Kopulationsapparat, bestehend aus dem zur Hälfte chitinisierten Bursalsegment mit seiner Apophyse (Ap I) und der linken Labie (L) mit ihrer Apophyse (Ap II). Merkwürdigerweise liegt eine unvollkommene bursa copulatrix (B. c.) auf der männlichen Seite. Gyn. M. Das Bursalsegment ist weiblich auf der Dorsalseite und links bis teilweise ventral, männlich rechts und teilweise ventral, die unvollständige Bursa selbst liegt auf der weiblichen Seite. Der eigentliche Kopulationsapparat scheint ähnlich zu sein wie bei Gyn. G. (Textfig. B). (Er wurde nur am ganzen Tier untersucht nicht nach Mazeration.) Aber es sind entweder zwei weibliche Labien oder eine Labie und ein Uncus vorhanden.

5. Die inneren Genitalien. Diese sind bei Gyn. G. höchst rudimentär (Textfig. C). Auf der weiblichen Körperseite fehlen innere Genitalien vollständig, auf der männlichen findet sich ein ductus ejaculatorius mit Samenblase und ein paar daran ansitzenden rudimentären Schläuchen, die wohl vas deferens und Kittdrüse darstellen. Von einer Geschlechtsdrüse konnte auch auf Schnitten keine Spur gefunden werden, doch 
wird vermutet, daß ein abnormer Hoden vorhanden war, der bei der Präparation platzte. Dagegen besitzt Gyn. M. links einen halben weiblichen und rechts einen halben männlichen Apparat (Textfig. D). Der Hoden ist ein gelblicher unregelmäBiger Körper, der die typischen vier Follikel zeigt (Ho). (Beim normalen ơ verwachsen die Hoden beider Körperseiten zu einer dunkel pigmentierten Kugel.) Vom vas deferens (V.d.) zweigt merkwürdigerweise ein Gang (*) zur vagina (Va) ab, der vielleicht aus dem rechten Eileiter entstanden ist. Das vas deferens geht in die Samenblase über (V.s.), an der in typischer Weise die Anhangsdrïse sitzt. Dann folgt der ductus ejaculatorius, an dem ein nnverständlicher Drüsenanhang hängt; der ductus mündet im Penis. Der weibliche Apparat zeigt die vier linken Ovarialschläuche $(\mathrm{Ov})$, wenn auch ein wenig rudimentär, von denen drei im ganzen 19 fertige Eier enthalten. Vagina (Va), receptaculum seminis (Rs), bursa copulatrix (B. c.) und Anhangsdrüse sind im wesentlichen wie bei einem normalen Weibchen entwickelt.

\section{Diskussion.}

Über das Wesen des Gyandromorphismus sind wir jetzt im wesentlichen orientiert: er ist die Folge abnormer Verteilung der Geschlechts-

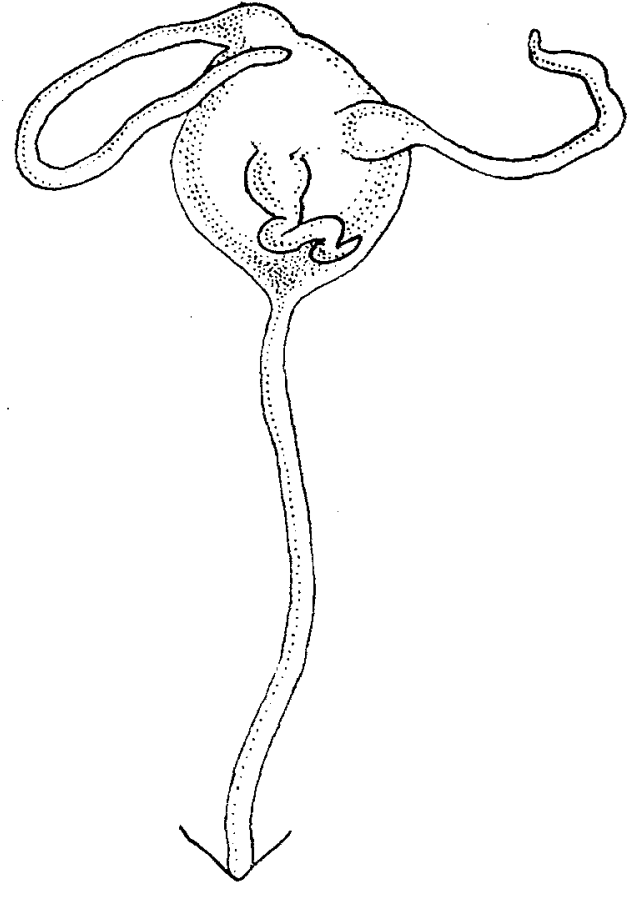

Textfig. C.

Innere Genitalien des Gyn. G. chromosomen, die es mit sich bringt, daß bestimmte Zellkomplexe weibliche, andere männliche Chromosomenkonstitution haben. Treten die abnormen Chromosomenverhältnisse so früh ein, daß die beiden ersten Furchungskerne 1 resp. $2 \mathrm{X}$-Chromosomen erhalten, so entsteht ein bilateraler Gynandromorph. (Literatur etc. s. bei Goldschmidt 1920b). Er muß dementsprechend genau in eine männliche und weibliche Hälfte halbiert sein. Bei unserm Objekt, dem Schwammspinner, kommen solche Stücke tatsächlich vor und sind äuch mehrfach beschrieben worden. 
Sie sind in allen Charakteren genau halbiert. Die hier beschriebenen beiden Stücke sind nun zweifellos echte bilaterale Gynandromorphe, denn Kopf, Antennen, Beine, Größe des Abdomens, Kopulationsapparat und (bei einem) innere Genitalien sind rechts völlig männlich und links völlig weiblich. Was nun zu erklären ist, ist, daß die Körper- und

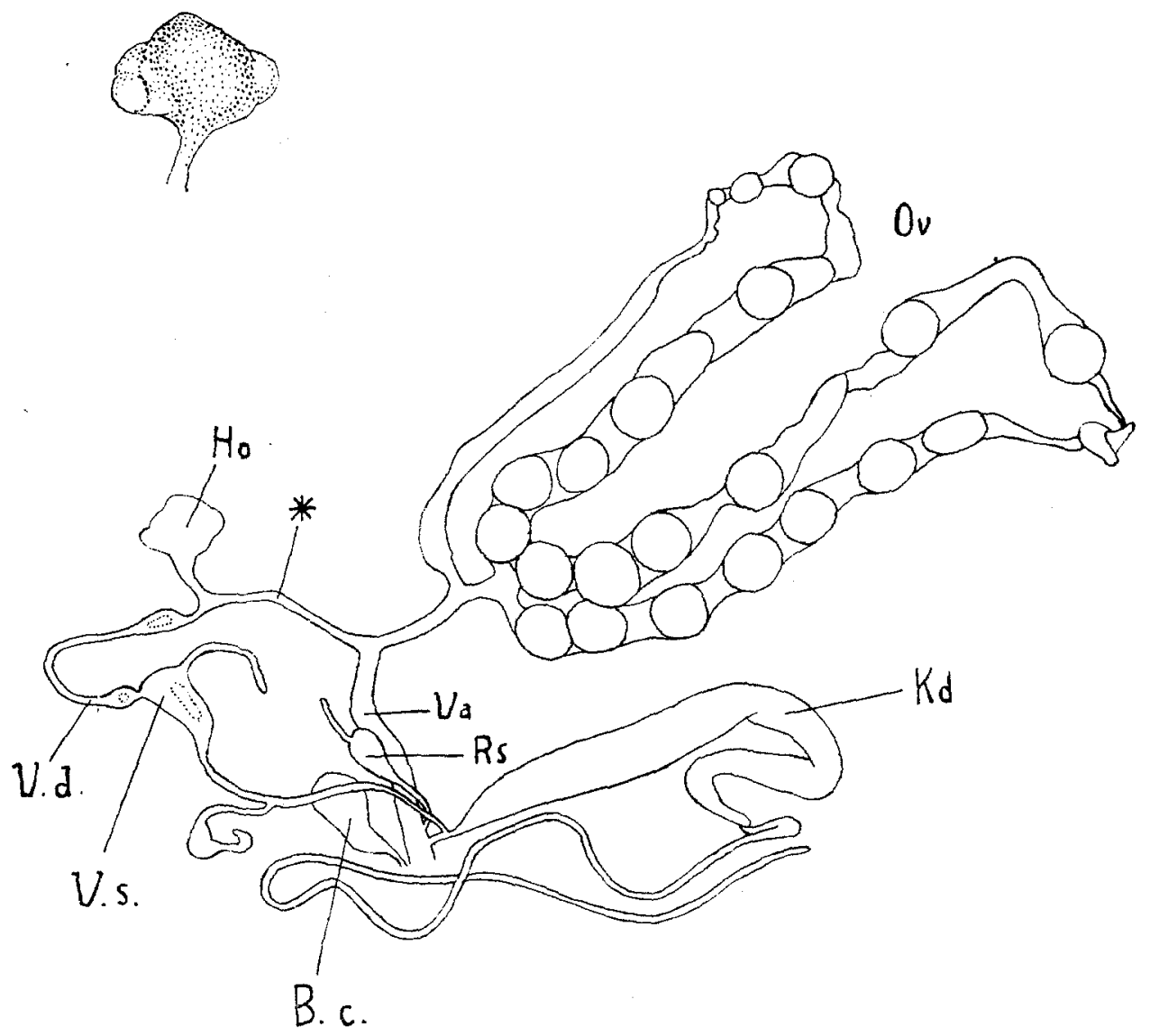

Textfig. D. Innere Genitalien des Gyn. M.

Flügelfarbe, auch Flügelform beim einen Stück männlich, dem andern weiblich mit leichten Einsprengungen der Charaktere des andern Geschlechts erscheinen. Zweitens ist zu erklären, daß der Kopulationsapparat nicht aus zwei Halbapparaten besteht.

Der letzte Punkt beansprucht ein beträchtliches entwicklungsmechanisches Interesse, wenn wohl auch seine Erklärung eine recht 
einfache ist. Bei dem genau untersuchten Organ des Gyn. G. ist der weibliche Teil tatsächlich genau ein halber weiblicher Apparat. Dagegen ist der männliche Teil fast vollständig. Die Entwicklungsgeschichte zeigt nun, daß der weibliche Kopulationsapparat, vor allem Labien und Apophysen symmetrische Bildungen darstellen, die sich rechts und links am Körperende differenzieren. So ist es nicht weiter merkwürdig, daß, wenn uur die linke Körperhälfte weiblich determiniert ist, auch nur die Hälfte des Apparates angelegt wird. Anders beim männlichen Kopulationsorgan. Seine Hauptteile, Penis und Valven, entwickeln sich aus einer medianen Tasche, den Heroldschen Organ, in der dann zwei Paar Zapfen sich erheben, die sich zu Valven und Penis formen. Es kann sich nun beim Gynandromorphen nicht gut eine halbe Tasche bilden. Da nun die Tasche die entwicklungsgeschichtliche Voraussetzung für die zwei Paar Zapfen darstellt, so genügt das Entstehen der Tasche, um auch in ihr die symmetrische Zapfenbildung hervorzurufen, somit die Valven beider Seiten und einen vollständigen Penis. Entwicklungsmechanisch ist der Vorgang der Entstehung eines Ganzembryo aus eiuer isolierten Furchungszelle zu vergleichen, ein Vergleich, der dadurch noch an Bedeutung gewinnt, daß der männliche Kopulationsapparat des Grnandromorphen nur halb so groß ist als ein normaler Apparat.

Ein viel verwickelteres Problem stellt abel die Frage der Flügelfärbung unserer Gynandromorphen dar; um sie diskutieren zu können, müssen zunächst ein paar Tatsachen aus einem ganz andersartigen Phänomenkomplex, der Intersexualität herangezogen werden. (Wegen der Einzelheiten s. Goldschmidt 1920a) Intersexualität unterscheidet sich von Gynandromorphismus dadurch, daß bei gleichbleibender weiblicher (weibliche Intersexualität) oder männlicher (männliche Intersexualität) Chromosomenkonstitution das Geschlecht des Individuums von einem bestimmten Moment seiner Entwicklung an in das entgegengesetzte umspringt. So wird der ganze Körper, nach Maßgabe des zur Zeit des Umschlages erreichten Entwicklungszustandes, von der Geschlechtsumwandlung betroffen. Mosaikbildungen, wie sie den Gynandromorphismus charakterisieren, sollten daher ausgeschlossen sein. Sie fehlen in der Tat, mit Ausnahme der Flügelfärbung. In der Reihe männlicher Intersexualität wandelt sich die männliche Flügelfärbung in die weibliche um, indem Flecke weiblicher Färbung im männlichen Flügel auftreten, deren Unfang sich mit fortschreitender Intersexualität vergrößert. In den Anfangsstadien finden sich ganz ähnliche Zustände, wie sie der Gyn. G. 
auf den Flügeln der weiblichen Seite zeigt. Bei weiblicher Intersexualität ist hingegen der Flügel meist ganz männlich gefärbt. Eine Ausnahme macht die Intersexualität, bei deren Hervorbringung eine ganz bestimmte Elternrasse (Gifu I) beteiligt ist. Bei dieser Serie verhält sich der Flügel intersexueller Weibchen genau wie die männliche Serie, zeigt also Mosaikbildungen. Die Anfangsstadien sehen aber ganz ähnlich ans wie die männliche Seite des Gyn. M., die Endstadien wie die weibliche Seite des Gyn. G.

Nun könnte man meinen, daß unsere Gynandromorphen vielleicht Mosaikbildungen nicht der beiden Geschlechter, sondern von weiblicher (resp. männlicher) Hälfte und intersexuellem Weibchen (resp. Männchen) seien. Dieser Gedanke, soweit er die beiden ganzen Körperhälften betrifft, ist abzulehnen. Denn dann müßte beim Gyn. G. die Seitë mit gescheckten Flügeln männliche Antennen und Kopulationsapparat besitzen, die für die betreffende Intersexualitätsstufe charakteristisch ist. Entsprechend müßte die rechte Seite des Gyn. M. weibliche Antennen, Kopulationsapparat und Gonade besitzen an Stelle des umgekehrten Verhaltens. Eine zweite Möglichkeit wäre die, daß die linke Seite des Gyn. G. nicht schwache männliche sondern starke weibliche Intersexualität repräsentierte; umgekehrt der Gyn. M. Auch dies ist anmöglich, da dann ebenfalls Antennen, Gonaden, Kopulationsapparate das entgegengesetzte Verhalten zeigen müßten.

So bietet sich als dritte Möglichkeit die, daß zwar beide Gynandromorphe richtige Halbseitenzwitter sind, rechts männlich, links weiblich, daß aber Flügel- und Körperfarbe allein eine Ausnahme machen, indem sie bei Gyn. G. auf der weiblichen Seite stark weiblich intersexuell sind, Gyn. M. auf der männlichen Seite stark männlich intersexuell. Nun kommen allerdings diese beiden Kombinationen unter den Bastarden aus den Rassen, deren Kreuzung die Gynandromorphen entstammen, nicht vor. Es wäre aber schließlich möglich, eine durch abnorme Teilungen entstehende Chromosomenkombination auszudenken, die dergleichen möglich machte. Aber eine solche Interpretation wird sehr unwahrscheinlich, wenn wir feststellen, daß die Flügelform gar nicht dazu paßt; sie müßte nämlich dann bei Gyn. M. rechts nicht weiblich sein, bei Gyn. G. links männlich. Noch weniger paßt aber das Verhalten des Abdomens dazu. Denn dies ist nach Wachstum, Form und Behaarung ganz oder vorwiegend das eines Halbseitenzwitters, während die Farbe im wesentlichen mit den Flügeln geht. So ist doch wohl eine andere Erklärung nötig. 
Die Erklärung muß nämlich anschließen an die Erklärung der ähnlichen Mosaikbildungen im intersexuellen Flügel. In den Untersuchungen über Intersexualität hat der eine von uns versucht, diese überaus schwierige Frage zu lösen: Die dort gefundenen Erklärungen haben sich aber durch neue Befunde als nicht haltbar erwiesen und nur ihre allgemeinste Form kann erhalten bleiben, nämlich, daß es sich um ein Teilproblem der noch so unklaren Entwicklungsphysiologie der Flïgelzeichnung handelt. Wir müssen deshalb hier auf eine vollständige Diskussion verzichten, die nur im Zusammenhang mit den Tatsachen der Intersexualität erfolgen kann, und verweisen anf eine in Vorbereitung befindliche Arbeit des einen Autors, die demnächst in dieser Zeitschrift erscheinen wird. Nur die folgenden Bemerkungen mögen hier Platz finden. 1. Die beiden Geschlechter des Schwammspinners unterscheiden sich durch verschiedene Entwicklungsgeschwindigkeiten, die auch noch auf die einzelnen Entwicklungsperioden verschiedenartig verteilt sind. 2. Die Rassen, aus deren Kreuzung Intersexe und auch unsere Gynandromorphe hervorgehen, verhalten sich in diesem Punkt sehr verschieden. 3. Im befruchteten Ei scheinen diese zeitlichen Verhältnisse bereits festgelegt $\mathrm{zu}$ sein. 4. Sie würden infolgedessen von einer bei der ersten Furchungsteilung eintretenden Abnormität, die den Halbseitenzwitter erzeugt, nicht beeinflußt werden. Wenn der Gyn. G. genetisch ein $\delta$, der Gyn. M. genetisch ein $ᄋ$ ist, so wäre die Differenzierungsgeschwindigkeit gewisser Vorgänge bei ihnen nur männlich resp. weiblich. 5. Es steht fest, daß bei der Entstehung der Flügelfärbung Differenzierungsgeschwindigkeiten der Schuppen eine große Rolle spielen. 6. So folgt, daß das eigentümliche Verhalten des gynandromorphen Flügel dadurch, allgemein ausgedrückt, zustande kommt, daß ein Gynandromorph auf der genetischen Grundlage eines bestimmten Geschlechts entsteht und die vorausgegangene Rassenkreuzung absonderliche Verhältnisse der Differenzierungsgeschwindigkeiten schafft, die in die Entwicklungsphysiologie der Flügel entscheidend eingreifen. Die Einzelausführung dieser Erklärung muß, wie gesagt, der sehr verwickelten Diskussion des Gesamtproblems vorbehalten bleiben.

Anmerkung bei der Korrektur. Inzwischen ist der eine der Verfasser (Goldschmidt) in den Besitz eines besonders interessanten Materials gekommen, das ihm gestatten wird, auf die hier aufgeworfenen Probleme ausführlich zurückzukommen. 
258 Goldschmidt und Machida. Utber zwei eigenartige Gynandromorphe usw.

\section{Zitierte Literatur.}

Cockayne, E. A., 1915, Gynapdromorphism and kindred problems. Journal of Genetics. Vol. 5.

Goldschmidt, R., $1920 \mathrm{a}$, Untersuchungen über Intersexualität. Zeitschr. f. ind. Abst.- u. Vererbgsl. 23.

- 1920 b, Mechanismus und Physiologie der Geschlechtsbestimmung. Berlin, Börntraeger, 1920.

Morgan, Th. and Bridges, C. F., 1919, The origin of gynandromorphs. Public. of Carnegie Inst. Washington. Nr. 278.

\section{Erklärung zu Tafel 3 .}

Fig. 1. Gyn. G. gespannt ohne Abdomen.

Fig. 2. Gyn. M. gespannt (Zeichnung).

Fig. 3. Gyn. G. Vorderende des lebenden Tieres.

Fig. 4. Gyn. M. Abdomen ventral (Zeichnung).

Fig. 5. Gyn. M.

Fig. 6. Gyn. G. von der Bauchseite, lebend.

Fig. 7. Gyn. G. von der Rückenseite, lebend, die Flügel zur Seite geschoben. 


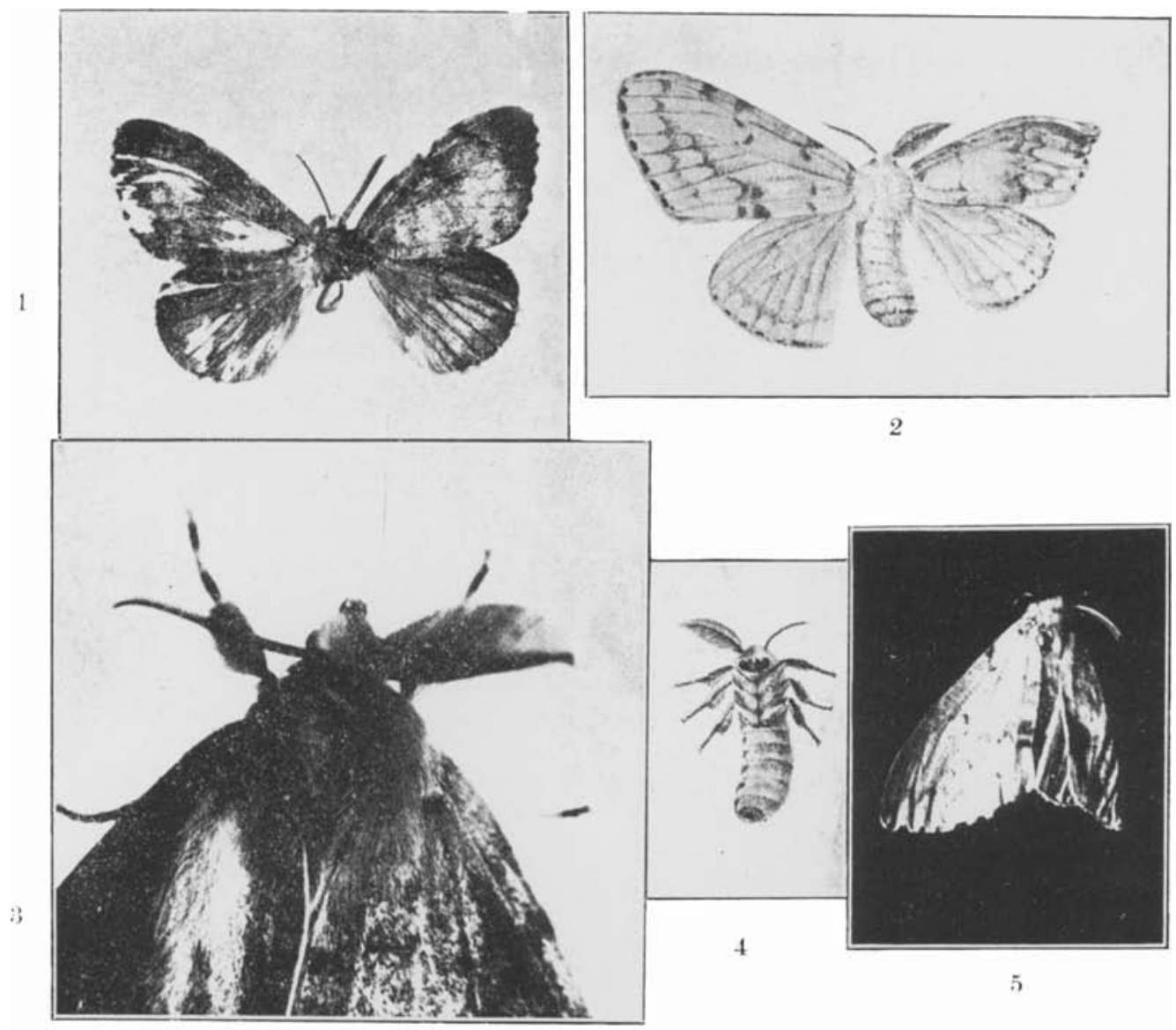

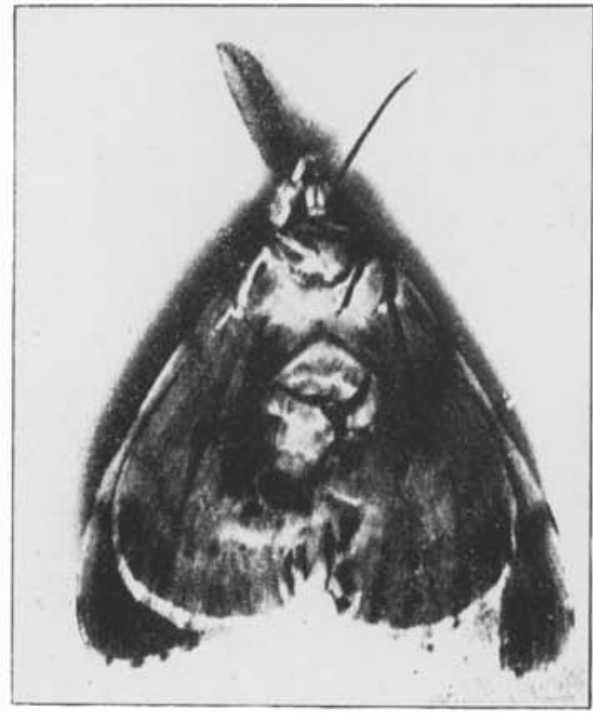

(6)

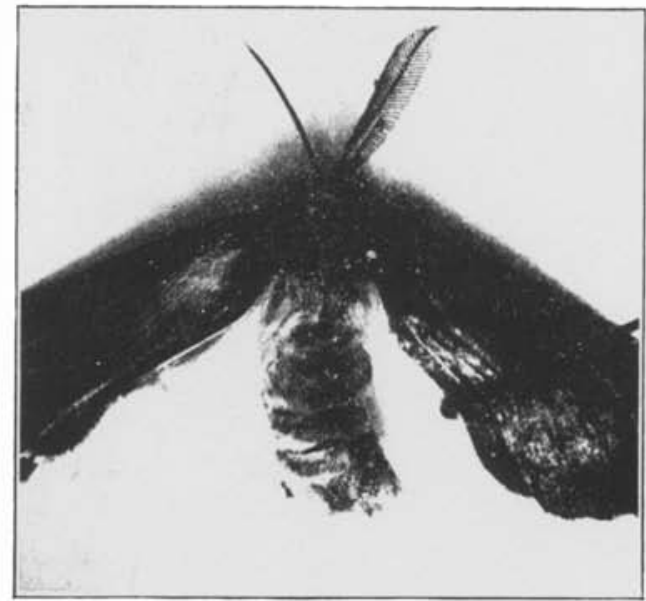

7

Goldschmidt u. Machida: Über zwei eigenartige Gynandromorphe usw. 